\title{
Ultrafast SEM
}

Petr Hlavenka $^{1 *}$, Erik Kieft ${ }^{2}$, Ernst Jan Vesseur ${ }^{2}$

1. Thermo Fisher Scientific, Brno, Czech Republic.

2. Thermo Fisher Scientific, Eindhoven, the Netherlands.

* Corresponding author: petr.hlavenka@thermofisher.com

Optical phenomena lie at the basis of applications in energy, lighting, analytical techniques, and information and communication technology. These phenomena take place on nanometer length scales and at ultrashort time scales. So far, light-based techniques have been used to study ultrafast optics, but they are limited in spatial resolution by diffraction. Electron microscopy is able to break the diffraction limit using a beam that has a deep sub-wavelength waist. By collecting the light emitted from an e-beam irradiated sample, the optical properties can thus be probed at much improved resolution. Recent advancements have also made it possible to produce short electron pulses. Carefully controlling the pulse length now allows the study of optical properties in the time domain. In this paper we present two techniques for creating such short electron pulses.

Technique 1: ultrafast blanker

Traditionally the electrostatic blanking systems on SEM served predominantly the electron lithography use-case, with pulses in the microsecond range. Recently we have exploited the technique of electrostatic blanking to generate sub-ns electron pulses without sacrificing the SEM versatility and performance [1]. The key improvements are the use of the conjugate blanking regime [2] together with the electrostatic deflector streaking the electron beam over the surface of a beam blocking aperture such that only angles close to optical axis allow the beam to pass, as depicted in figure 1a). The set of electro-optical elements of Thermo Fisher field emission (FEG) class of SEM systems can be set such that the beam cross-over is precisely positioned to the centre of deflection plates. This way the small residual beam movement caused by the blanker deflection is almost fully compensated in the focus plane at a specimen. In this optical regime the Quattro SEM can achieve resolution better than $20 \mathrm{~nm}$ at $\mathrm{nA}$ probe current, as demonstrated by the image in figure 1c). The image of tin balls has been acquired at $5 \mathrm{keV}$ with deflection electrodes driven with $10 \mathrm{MHz}$ sinus wave and amplitude allowing for 50\% transmitted beam current. The corresponding DC probe current was $0.8 \mathrm{nA}$. On a Helios FIB-SEM we achieved even better resolution where at $2 \mathrm{keV}$ landing energy we measured a resolution of $3.1 \mathrm{~nm}$ at analogous blanker setting.

The temporal profile of the electron pulse has been measured directly by using a streak camera with deflection plates mounted on the polepiece and a Timepix direct electron camera mounted at $50 \mathrm{~mm}$ distance. A saw-shaped voltage signal with $2 \mathrm{kV}$ amplitude has been applied to the deflection plates of the streak camera. The intensity of the electron pulse trace on the camera is thus directly correlated with temporal pulse profile depicted in figure $1 \mathrm{~b}$ ), where a pulse of $5 \mathrm{keV}$ electrons have duration below $500 \mathrm{ps}$.

The most remarkable benefit of our setup is its versatility. The switch from an SEM with uncompromised performance to a sub-ns high-resolution electron pulse prober requires only changing the optical aperture and loading the pre-sets for alternative optical setup.

Technique 2: photoemission 
The method of creating pulsed photoemission from a FEG source, for Ultrafast pump-probe applications, has been explored at Caltech [3]. On Quattro SEMs modified for this purpose, laser access is provided towards the gun region for side-illumination of the Schottky FEG tip, while the microscope can also still be operated in normal field emission mode without compromise to its performance. Changing the source parameters from normal field emission conditions to pulsed operation leads to some inherent loss of beam brightness; and a balance needs to be found between total signal per pulse on one hand, and spatial resolution on the other. Compared to normal operation this can be realized by lowering the extractor voltage and/or increasing the suppressor bias, to achieve a more collimated electron beam and more electrons in the pulse reaching the specimen, at the cost of some reduction of beam coherence. However, photoemission offers the potential of electron pulse durations on the sub-ps timescale, and straightforward synchronization with arbitrary precision in pump-probe experiments, a benefit not currently available from any other method.

In conclusion, advances in time-resolved SEM have made ultrashort electron pulses more accessible to researchers and scientist in a broad range of fields. We expect that the technique will stimulate results in fields such as luminescent materials, photovoltaics, opto-electronics, geoscience, coatings, life science, and (quantum) communication [4].

References:

[1] S Meuret et al., Ultramicroscopy 197 (2019), p. 28.

[2] M Gesley, Review of Scientific Instruments 64 (1993), p. 3169.

[3] D Yang, OF Mohammed and AH Zewail, PNAS 107 (2010), 14993.

[4] The authors acknowledge funding from Technology Agency of the Czech Republic, TE01020118.

a)

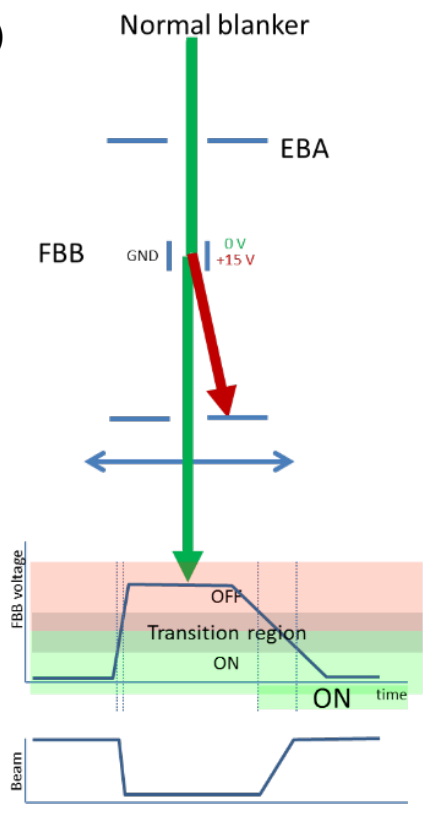

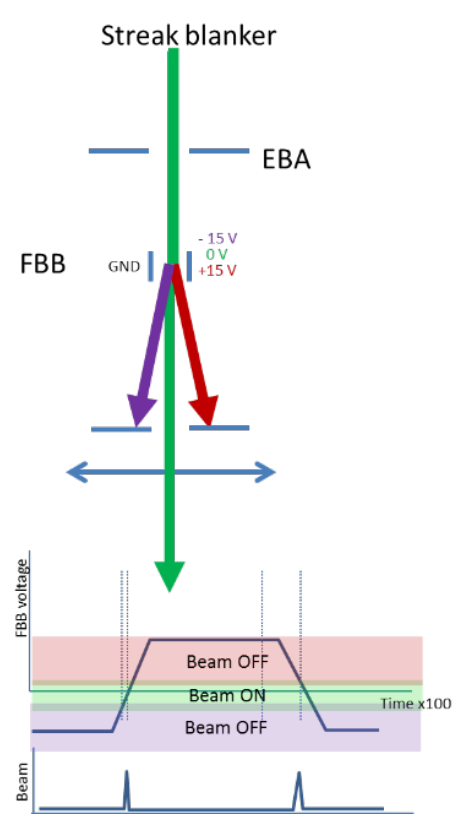

b)

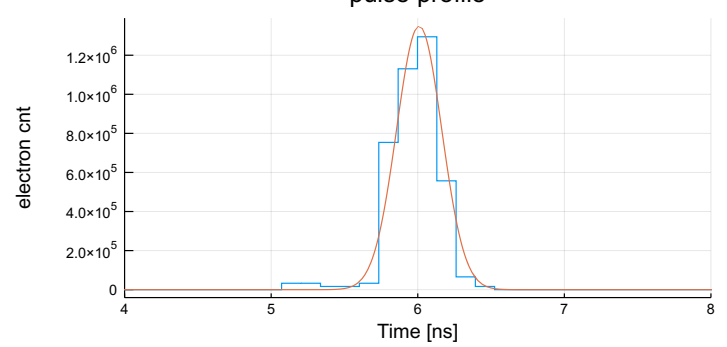

c)

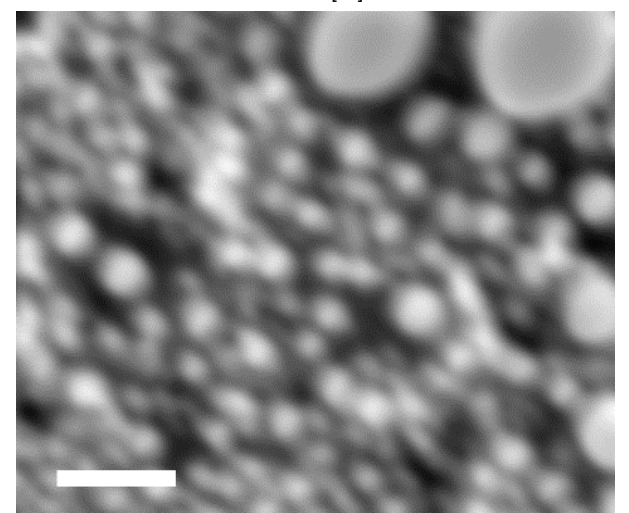

Figure 1. a) Scheme of short pulse generation by beam streaking, b) the temporal profile of an electron pulse in a streak-through mode at $5 \mathrm{keV}$ with FWHM of $364 \mathrm{ps}$, c) image of tin balls obtained at the same conditions in strike-through regime with 50\% beam-on time, scalebar $200 \mathrm{~nm}$. 